Review began 11/01/2021 Review ended 11/29/2021 Published 11/30/2021

๑) Copyright 2021

Shaikh et al. This is an open access article distributed under the terms of the Creative Commons Attribution License CC-BY 4.0. which permits unrestricted use, distribution, and reproduction in any medium, provided the original author and source are credited.

\section{Brain Metastasis in a Young Patient: Consider the Rectum}

\author{
Abdullah S. Shaikh ${ }^{1}$, Ravi Pavurala ${ }^{2}$, Eric Gou ${ }^{3}$ \\ 1. Internal Medicine, University of Texas Medical Branch, Houston, USA 2. Gastroenterology and Hepatology, \\ University of Texas Medical Branch, Galveston, USA 3. Gastroenterology, University of Texas Medical Branch, \\ Galveston, USA
}

Corresponding author: Abdullah S. Shaikh, asshaikh@utmb.edu

\begin{abstract}
Approximately $20 \%$ of patients with newly diagnosed colorectal cancer present with distant metastatic disease. Brain metastasis from colorectal cancer is uncommon and usually associated with metachronous metastases in other organs. We describe a rare case of a 49-year-old patient presenting with headaches and left-sided weakness found to have a solitary brain metastasis from primary rectal cancer. Primary rectal cancer, young age, lung and liver metastases, and KRAS mutation are risk factors associated with brain metastases in patients with colorectal cancer. Intracranial imaging should be considered as part of the workup in the staging of colorectal cancer in patients who are at high risk of brain metastasis.
\end{abstract}

Categories: Neurology, Gastroenterology, Oncology

Keywords: brain tumor, brain, metastatic colo-rectal cancer, metastatic, colorectal cancer

\section{Introduction}

Colorectal cancer is the third most common cancer in the United States [1] and while an increasing number of patients are now being diagnosed through colorectal cancer screening, the overwhelming majority of patients are still being diagnosed after the onset of symptoms [2, 3]. Typical presenting symptoms include a change in bowel habits, rectal bleeding, a rectal or abdominal mass, or iron deficiency anemia [4]. Moreover, approximately $20 \%$ of patients with newly diagnosed colorectal cancer present with distant metastatic disease [5]. The most common sites of metastasis are liver, lung, peritoneum and lymph nodes [6]. Brain metastasis from colorectal cancer is uncommon and usually associated with metachronous metastases in other organs. We describe a rare case of a 49-year-old patient presenting with headaches and left-sided weakness found to have a solitary brain metastasis from primary rectal cancer.

\section{Case Presentation}

A 49-year-old Caucasian male with a history of tobacco and alcohol abuse presented with worsening headaches, unintentional weight loss, and left-hand weakness. He reported a one-month history of worsening and persistent headaches as well as $30 \mathrm{lbs}$. of unintentional weight loss. He then developed left hand weakness approximately 2 weeks prior to presentation; he also reported increased left hand dyscoordination, which led him to dropping things often. He was taken to the emergency room by a coworker when he became unresponsive. His neurological exam on presentation was significant for $4 / 5$ strength as well as a subtle drift in his left upper extremity. The rest of his physical exam was unremarkable. MRI brain showed a $4.4 \times 4.0 \times 3.7 \mathrm{~cm}$ right frontal mass with associated $1.3 \mathrm{~cm}$ right to left midline shift (Figure 1); the patient did not undergo positron emission tomography (PET) scan. 


\section{Cureus}

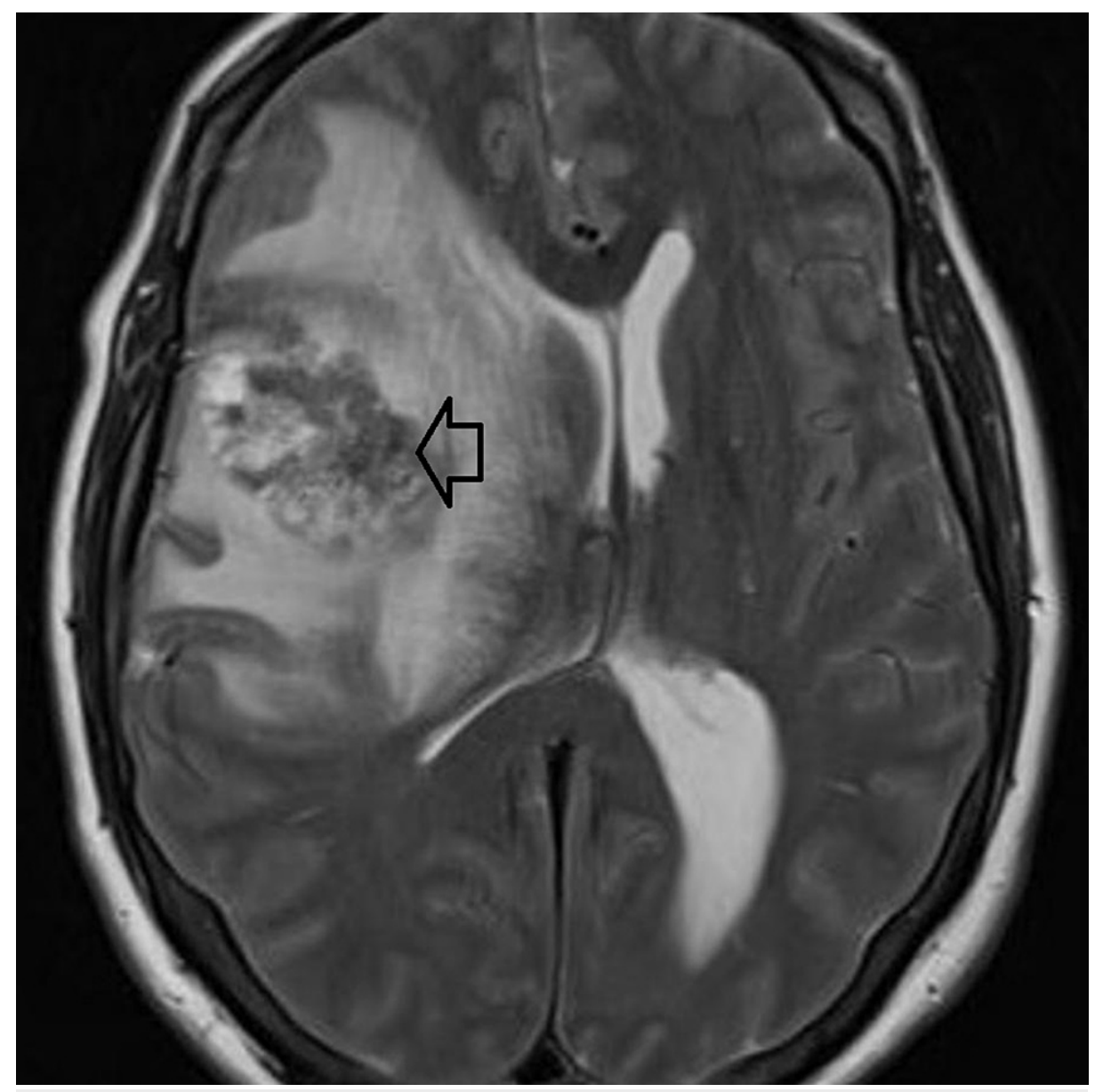

FIGURE 1: MRI of brain with and without contrast showing right lateral frontal intra-axial heterogenous enhancing mass measuring $4.4 \times 4.0 \times$ $3.7 \mathrm{~cm}$ with surrounding edema and right to left midline shift

The patient was admitted to the neurosurgery service and underwent a right craniotomy due to initial suspicion of a high-grade glioma. However, pathology of the resected mass showed adenocarcinoma with an immunohistochemical profile most consistent with colorectal primary malignancy. Further review of systems revealed eight months of non-bloody diarrhea associated with fecal urgency. He did not have a family history of colorectal cancer in any first-degree relative. Carcinoembryonic antigen was elevated at 4.6 $\mathrm{ng} / \mathrm{mL}$. CT imaging of the chest, abdomen and pelvis showed ill-defined, nodular wall thickening of the rectum and sigmoid colon, sub-centimeter presacral and lower para-aortic lymph nodes and no other distant metastatic lesions. Flexible sigmoidoscopy demonstrated a partially obstructing rectal mass (Figure 2) with biopsies consistent with rectal adenocarcinoma. Immunohistochemistry showed microsatellite stability and molecular studies for KRAS, NRAS, and BRAF mutations were negative. He was referred to oncology and a repeat MRI brain did not show any evidence of any new intracranial metastases. The patient is awaiting systemic palliative chemotherapy with FOLFOX and bevacizumab. 


\section{Cureus}

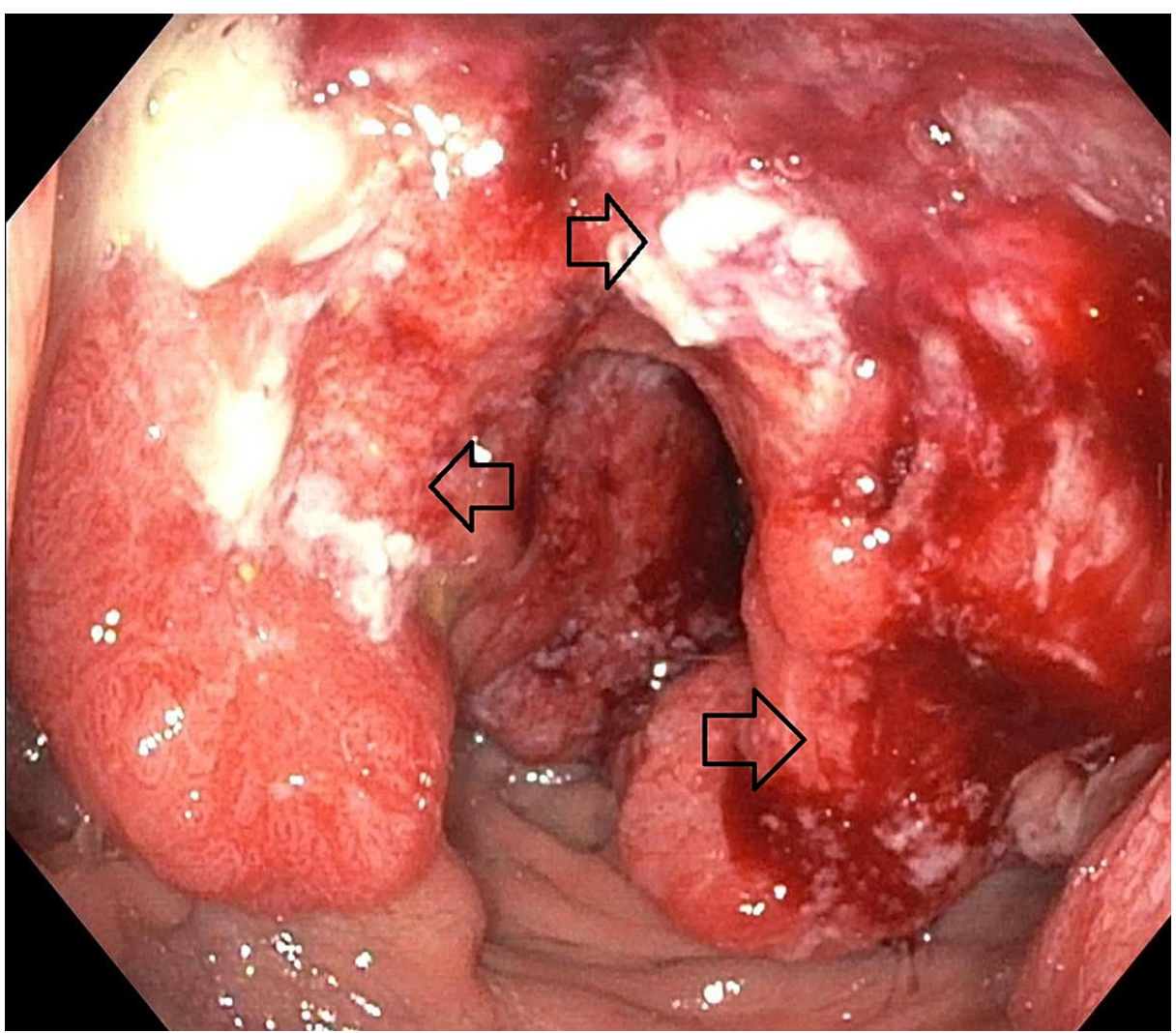

FIGURE 2: Endoscopic view of rectum showing fungating and polypoid circumferential mass with luminal narrowing extending into the anus

\section{Discussion}

The median age of diagnosis of rectal cancer is 63 years for both men and women with only $12 \%$ of cases of colorectal cancer occurring in patients under the age of 50 [7]. Moreover, the likelihood of a rectal cancer having a single metastasis to the nervous system is approximately $3 \%$ compared to $81 \%$ likelihood of a single metastasis to the liver $(62 \%)$ or thorax (19\%) as based on a multivariable logistic regression analysis of approximately 3,000 patients with rectal cancer with a single metastasis [6]. Another systematic review found that the incidence of a brain metastasis from colorectal cancer is extraordinarily uncommon with an incidence of approximately 0.6 to $3.2 \%$ and is usually an indicator of late-stage colorectal cancer [8]. Brain metastasis is much more common in other cancers such as lung cancer, breast cancer, testicular cancer, and melanoma with an incidence ranging from 10-50\% depending on the type of cancer [8].

Our patient's presentation is unique in that he presented at a young age with rectal cancer and a solitary metastasis to the brain. The incidence of brain metastasis at the initial diagnosis of colorectal cancer is $1.38 \%$ with a median survival of four months [9]. Moreover, a recent metanalysis and systemic review of brain metastasis in colorectal cancer recommended MRI imaging of the brain if a patient with colorectal cancer had any neurological symptoms or risk factors for brain metastases such as the presence of lung metastases, KRAS mutation, high carcinoembryonic antigen (CEA) level, or rectal cancer [10]. The rising incidence of early-onset colorectal cancer, which are characterized by poorer cell differentiation, more advanced stage at diagnosis, as well as more often having a left-sided colon primary tumor [11], is one of the primary reasons the U.S. Preventative Task Force updated their recommendation to start colorectal cancer screening from age 45 [12], instead of waiting till age 50 .

There is an increasing concern of microsatellite instability leading to proximal colon cancer with RAS and BRAF mutations found in approximately $52 \%$ [13] and $10 \%$ [14] of colorectal cancer, respectively. While our patient did not have evidence of microsatellite instability or a proximal colon cancer, RAS and BRAF mutation testing has been shown to have prognostic and predictive value in metastatic colorectal cancer. Treatment with anti-epidermal growth factor receptor (EGFR) antibodies has been shown to be less effective in patients with KRAS mutations [15] and patients with metastatic colorectal cancer and either KRAS or BRAF mutations have been shown to have worsened outcomes [16].

\section{Conclusions}

In conclusion, brain metastases are the most common intracranial neoplasm and typically arise from 
melanoma, lung, and breast cancers. Brain metastases from gastrointestinal cancers are uncommon and occur in less than $4 \%$ of patients with colorectal cancer. Primary rectal cancer, young age, lung and liver metastases, and KRAS mutation are risk factors associated with brain metastases in patients with colorectal cancer. Intracranial imaging should be considered as part of the workup in the staging of colorectal cancer in patients who are at high risk of brain metastasis.

\section{Additional Information \\ Disclosures}

Human subjects: Consent was obtained or waived by all participants in this study. Conflicts of interest: In compliance with the ICMJE uniform disclosure form, all authors declare the following: Payment/services info: All authors have declared that no financial support was received from any organization for the submitted work. Financial relationships: All authors have declared that they have no financial relationships at present or within the previous three years with any organizations that might have an interest in the submitted work. Other relationships: All authors have declared that there are no other relationships or activities that could appear to have influenced the submitted work.

\section{References}

1. Siegel RL, Miller KD, Jemal A: Cancer statistics, 2020. CA Cancer J Clin. 2020, 70:7-30. 10.3322/caac.21590

2. Moreno CC, Mittal PK, Sullivan PS, et al.: Colorectal cancer initial diagnosis: screening colonoscopy, diagnostic colonoscopy, or emergent surgery, and tumor stage and size at initial presentation. Clin Colorectal Cancer. 2016, 15:67-73. 10.1016/j.clcc.2015.07.004

3. Moiel D, Thompson J: Early detection of colon cancer-the kaiser permanente northwest 30-year history: how do we measure success? Is it the test, the number of tests, the stage, or the percentage of screendetected patients?. Perm J. 2011, 15:30-8. 10.7812/tpp/11-128

4. Thompson MR, O'Leary DP, Flashman K, Asiimwe A, Ellis BG, Senapati A: Clinical assessment to determine the risk of bowel cancer using Symptoms, Age, Mass and Iron deficiency anaemia (SAMI). Br J Surg. 2017, 104:1393-404. 10.1002/bjs.10573

5. Siegel RL, Miller KD, Jemal A: Cancer statistics, 2016. CA Cancer J Clin. 2016, 66:7-30. 10.3322/caac.21332

6. Riihimäki M, Hemminki A, Sundquist J, Hemminki K: Patterns of metastasis in colon and rectal cancer. Sci Rep. 2016, 6:29765. 10.1038/srep29765

7. Siegel RL, Miller KD, Goding Sauer A, et al.: Colorectal cancer statistics, 2020. CA Cancer J Clin. 2020, 70:145-64. 10.3322/caac.21601

8. Christensen TD, Spindler KL, Palshof JA, Nielsen DL: Systematic review: brain metastases from colorectal cancer--Incidence and patient characteristics. BMC Cancer. 2016, 16:260. 10.1186/s12885-016-2290-5

9. Lei S, Ge Y, Tian S, et al.: Colorectal cancer metastases to brain or bone and the relationship to primary tumor location: a population-based study. J Gastrointest Surg. 2020, 24:1833-42. 10.1007/s11605-01904308-8

10. Müller S, Köhler F, Hendricks A, et al.: Brain metastases from colorectal cancer: a systematic review of the literature and meta-analysis to establish a guideline for daily treatment. Cancers (Basel). 2021, 13:900. 10.3390/cancers13040900

11. Mauri G, Sartore-Bianchi A, Russo AG, Marsoni S, Bardelli A, Siena S: Early-onset colorectal cancer in young individuals. Mol Oncol. 2019, 13:109-31. 10.1002/1878-0261.1241'

12. Davidson KW, Barry MJ, Mangione CM, et al.: Screening for colorectal cancer: US Preventive Services Task Force recommendation statement. JAMA. 2021, 325:1965-77. 10.1001/jama.2021.6238

13. Cox AD, Fesik SW, Kimmelman AC, Luo J, Der CJ: Drugging the undruggable RAS: mission possible?. Nat Rev Drug Discov. 2014, 13:828-51. 10.1038/nrd4389

14. Seligmann JF, Fisher D, Smith CG, et al.: Investigating the poor outcomes of BRAF-mutant advanced colorectal cancer: analysis from 2530 patients in randomised clinical trials. Ann Oncol. 2017, 28:562-8. 10.1093/annonc/mdw645

15. Dahabreh IJ, Terasawa T, Castaldi PJ, Trikalinos TA: Systematic review: anti-epidermal growth factor receptor treatment effect modification by KRAS mutations in advanced colorectal cancer. Ann Intern Med. 2011, 154:37-49. 10.7326/0003-4819-154-1-201101040-00006

16. Modest DP, Ricard I, Heinemann V, et al.: Outcome according to KRAS-, NRAS- and BRAF-mutation as wel as KRAS mutation variants: pooled analysis of five randomized trials in metastatic colorectal cancer by the AIO colorectal cancer study group. Ann Oncol. 2016, 27:1746-53. 10.1093/annonc/mdw261 